\title{
Equivalent linear change in cognition between individuals with bipolar disorder and healthy controls over 5 years
}

\author{
Kelly A Ryan $^{1}$ (D) | Shervin Assari ${ }^{1}$ (D) | Kaley Angers ${ }^{1}$ | David F Marshall $^{1}$ | \\ Kristin Hinrichs $^{1,2}$ | Rebecca Easter ${ }^{1}$ | Pallavi Babu ${ }^{1}$ | Bethany D Pester ${ }^{1}$ | \\ Scott A Langenecker ${ }^{3}$ | Melvin G McInnis ${ }^{1}$
}

\author{
${ }^{1}$ Department of Psychiatry, University of \\ Michigan, Ann Arbor, MI, USA \\ ${ }^{2}$ SSM Health Rehabilitation Hospital, \\ Bridgeton, MO, USA \\ ${ }^{3}$ Department of Psychiatry, University of \\ Illinois at Chicago, Chicago, IL, USA

\section{Correspondence} \\ Kelly A. Ryan, 2101 Commonwealth Blvd, \\ Suite C, Ann Arbor, MI 48105, USA. \\ Email: karyan@umich.edu \\ Funding information \\ This research was supported by the Heinz \\ C. Prechter Bipolar Research Fund at the \\ University of Michigan Depression Center \\ and the Richard Tam Foundation (KR, SA, \\ $\mathrm{BP}, \mathrm{KH}, \mathrm{DM}, \mathrm{AB}, \mathrm{KA}, \mathrm{DS}, \mathrm{MK}$ and $\mathrm{MM})$, and \\ grant support from the National Center for \\ Advancing Translational Sciences of the \\ National Institutes of Health under Award \\ Number 2KL2TR000434 (KR).
}

\begin{abstract}
Objectives: Cognitive dysfunction is a key feature of bipolar disorder (BD). However, not much is known about its temporal stability, as some studies have demonstrated a neurodegenerative model in BD while others have shown no change in cognitive functioning over time. Building upon our prior work, which examined the natural course of executive functioning, the current study aimed to investigate the natural course of memory, emotion processing, and fine motor dexterity over a 5-year period in BD and healthy control $(\mathrm{HC})$ samples.

Methods: Using a 5-year longitudinal cohort, 90 individuals with BD and $17 \mathrm{HCs}$ were administered a battery of neuropsychological tests at study baseline and at 1 and 5 years after study entry that captured four areas of cognitive performance: visual memory, auditory memory, emotion processing, and fine motor dexterity.

Results: Latent growth curve modeling showed no group differences in the slopes of any of the cognitive factors between the BD and $\mathrm{HC}$ groups. Age at baseline was negatively associated with visual memory, emotion processing, and fine motor dexterity. Education level was positively associated with auditory and visual memory and fine motor. Female gender was negatively associated with emotion processing.

Conclusions: Extending our prior work on longitudinal evaluation of executive functioning, individuals with BD show similar linear change in other areas of cognitive functioning including memory, emotion processing, and fine motor dexterity as compared to unaffected HCs. Age, education, and gender may have some differential effects on cognitive changes.

KEYWORDS

affective disorders, bipolar disorder, cognition, neurodegenerative
\end{abstract}

\section{1 | INTRODUCTION}

Cognitive dysfunction is considered a core feature of bipolar disorder (BD) that is apparent during acute mood states as well as during periods of euthymia. ${ }^{1-5}$ However, the temporal stability of this cognitive dysfunction is less clear, as few longitudinal studies have found consistent results. Several studies have shown progressive decline in functioning, ${ }^{6-8}$ supporting a neurodegenerative model in BD, whereas others have shown no change in cognitive functioning over time. ${ }^{9-14}$

In a cross-sectional study we conducted in 2013 , using a large sample of individuals with $\mathrm{BD}$, we found that those with BD performed worse than unaffected healthy controls (HCs) in four different areas of executive functioning, ${ }^{4,15}$ consistent with prior literature. ${ }^{16,17}$ In the 5 -year follow-up of the same cohort, the linear change on measures 
TAB LE 1 Demographic characteristics and cognitive performance factor scores for the bipolar and healthy control groups.

\begin{tabular}{|c|c|c|c|c|c|c|c|}
\hline \multirow[b]{2}{*}{ Baseline } & \multicolumn{4}{|c|}{ Baseline } & \multirow[b]{2}{*}{ Effect size $e^{c}$} & \multicolumn{2}{|c|}{1 year } \\
\hline & Bipolar $n=90$ & $\begin{array}{l}\text { Healthy } \\
\text { controls } n=17\end{array}$ & $t$ & $P$ & & Bipolar $n=90$ & $\begin{array}{l}\text { Healthy } \\
\text { controls } n=17\end{array}$ \\
\hline Age & $42.06(11.30)$ & $35.88(15.48)$ & -1.94 & .06 & 0.46 & - & - \\
\hline Education & $15.53(2.18)$ & $16.71(2.02)$ & 2.05 & .04 & -0.56 & - & - \\
\hline \multicolumn{8}{|l|}{ Gender ${ }^{a}$} \\
\hline$\%$ female & 74.40 & 76.50 & 0.03 & .86 & 0.02 & - & - \\
\hline$V_{\text {Verbal intelligence }}^{b}$ & $12.49(2.81)$ & $13.18(3.12)$ & 0.91 & .37 & -0.23 & - & - \\
\hline HRDS & $8.23(5.70)$ & $1.97(2.20)$ & -4.45 & $<.001$ & 1.45 & $7.74(5.94)$ & $1.60(2.15)$ \\
\hline YMRS & $2.59(3.47)$ & $0.29(0.59)$ & -2.71 & .01 & 0.92 & 2.64 (3.97) & $0.86(1.70)$ \\
\hline Auditory memory & $-0.36(0.82)$ & $-0.22(0.84)$ & 0.62 & .54 & -0.17 & $-0.33(0.86)$ & $0.02(1.06)$ \\
\hline Visual memory & $-0.52(1.17)$ & $0.49(1.10)$ & 3.31 & .001 & -0.89 & $-0.23(1.20)$ & $0.61(0.98)$ \\
\hline Fine motor & $-0.82(1.02)$ & $0.28(0.78)$ & 4.21 & $<.001$ & -1.21 & $-0.61(1.10)$ & $0.45(0.91)$ \\
\hline Emotion processing & $-0.44(1.64)$ & 0.19 (0.69) & 1.30 & .20 & -0.50 & $-0.33(1.63)$ & $0.40(0.81)$ \\
\hline
\end{tabular}

Data are presented as mean (SD).

HRDS, Hamilton Rating Scale for Depression; YMRS, Young Mania Rating Scale.

${ }^{\mathrm{a} C}$ Chi-square analyses.

bWASI IQ, Wechsler Abbreviated Scale of Intelligence Vocabulary scaled score.

${ }^{c}$ Cohen's $d$ or Cramer's V.

of executive functioning among those with BD was no different from the change for $\mathrm{HCs}^{15}$ This suggests that the longitudinal course of executive functioning may not be dependent on having a BD diagnosis. Further, these results were interpreted to suggest that the executive functioning deficits in BD are not age-accelerated, age-compounded, or neurodegenerative, extending earlier work using smaller samples. ${ }^{1,18,19}$

We extend our prior work ${ }^{15}$ by now investigating other areas of cognition commonly known to be deficient in $\mathrm{BD}$, notably the areas of memory, emotion processing, and fine motor dexterity. These cognitive areas, particularly memory, may be more appropriate to examine in order to determine disease-related progressive change, as one longitudinal study found that memory was the only cognitive area subject to change over time in $\mathrm{BD} .^{3}$ Therefore, our main objective was to examine the longitudinal trajectory of verbal and visual memory, emotion processing, and fine motor dexterity over 5 years in a large sample of individuals with $\mathrm{BD}$ who were being followed in the Prechter Longitudinal Study of BD. ${ }^{4,20}$ Similar to our prior work, ${ }^{15}$ we used a statistical approach, latent growth curve modeling (LGCM), that offers many advantages over traditional methods for longitudinal analyses ${ }^{21}$ in that it can examine nonlinear relationships in cognitive change, correlate measurement errors related to each cognitive variable over time, correlate trajectories with each other, and account for any missing data. Consistent with our prior findings and those of others, we hypothesized that memory, emotion processing, and fine motor dexterity would be systematically worse in the BD group as compared to the $\mathrm{HC}$ group at baseline (study entry). Further, based on similar trajectories of executive functioning across 5 years in this sample, ${ }^{15}$ we expected there would be similar linear changes in memory, emotion processing, and fine motor dexterity over 5 years, indicating that these cognitive deficits in BD are likely not age-accelerated, agecompounded, or neurodegenerative. ${ }^{22}$

\section{2 | MATERIALS AND METHODS}

\section{1 | Subjects}

Participants were enrolled in the Prechter Longitudinal Study of BD, an observational cohort study gathering phenotypic and biological data, at the University of Michigan. The University of Michigan institutional review board (IRB) approved this study, all participants gave informed consent, and each participant received incentive payment for participation in the longitudinal study. Recruitment for the study occurred through advertisements in community mental health centers, in an outpatient specialty psychiatric clinic, in an inpatient psychiatric clinic, in local newspapers, at community outreach events, and on the web. Participants who were enrolled in the longitudinal study from 2005 to 2008 and had 5-year follow-up data were used for this study. This included HCs and individuals who had a diagnosis of BD. Out of the 264 participants who had been enrolled long enough to complete a 5-year follow-up visit, 108 completed the 5year neuropsychological re-testing and thus were included in this study's main analyses. Ninety-one of those individuals had BD (bipolar disorder type I [BD I], 80 participants; bipolar disorder type II [BD II], nine participants; bipolar disorder not otherwise specified [BD-NOS], two participants), and 17 were HCs. Eight HCs who completed re-testing were excluded from analyses as their diagnoses had changed (four received a new diagnosis of major depressive disorder, one depression NOS, one post-traumatic stress disorder [PTSD], one BD II, and one BD I). Notably, there was no significant difference between these participants' baseline and 5-year neuropsychological scores. Specific comparisons between those with BD and HCs who completed or did not complete the 5-year follow-up testing are presented in our prior work. ${ }^{15}$ Overall there were no differences 


\begin{tabular}{|c|c|c|c|c|c|c|c|}
\hline \multirow[b]{2}{*}{$t$} & \multirow[b]{2}{*}{$P$} & \multirow[b]{2}{*}{ Effect size $^{c}$} & \multicolumn{4}{|c|}{5 year } & \multirow[b]{2}{*}{ Effect size $^{c}$} \\
\hline & & & Bipolar n=90 & $\begin{array}{l}\text { Healthy controls } \\
n=17\end{array}$ & $t$ & $P$ & \\
\hline- & - & & - & - & - & - & \\
\hline- & - & & - & - & - & - & \\
\hline- & - & & - & - & - & - & \\
\hline- & - & & - & - & - & - & \\
\hline-4.20 & $<.001$ & 1.37 & 7.54 (5.33) & $1.00(1.17)$ & -5.02 & $<.001$ & 1.69 \\
\hline-1.65 & .10 & 0.58 & 3.04 (3.75) & $0.76(1.52)$ & -2.46 & .02 & 0.80 \\
\hline 1.49 & .14 & -0.36 & $-0.27(0.79)$ & $-0.05(1.07)$ & 1.00 & .32 & -0.23 \\
\hline 2.72 & .01 & -0.77 & $0.19(1.21)$ & $1.14(1.06)$ & 3.02 & .003 & -0.84 \\
\hline 3.84 & $<.001$ & -1.05 & $-1.06(1.16)$ & $0.04(0.93)$ & 3.60 & $<.001$ & -1.04 \\
\hline 1.75 & .08 & -0.57 & $-0.12(0.92)$ & $0.11(1.00)$ & 0.92 & .36 & -0.24 \\
\hline
\end{tabular}

between the "completers" and "non-completers" in terms of clinical status and clinical variables.

All participants were evaluated at study baseline to confirm diagnoses using the Diagnostic Interview for Genetic Studies (DIGS). ${ }^{23}$ To come to a consensus on the most accurate diagnosis for the participants, the DIGS information and medical records were considered by two authors during a best estimate process. If participants had active substance use or a neurological disease at the time of enrollment, they were excluded. Clinician-administered measures (Hamilton Rating Scale for Depression [HRDS] ${ }^{24}$; Young Mania Rating Scale $\left[\right.$ YMRS] ${ }^{25}$ ) were given by trained staff, who were supervised by a study clinician, to rate mood symptoms for each participant during neuropsychological testing at baseline, year 1 , and year 5 (Table 1). Those with BD had a range of mood symptoms. Each participant's medication classes and composite load score were determined with methods adapted from other groups, ${ }^{26-30}$ in which higher scores represent a larger medication burden. To describe our BD sample, clinical variables obtained from the DIGS interview are listed in Supporting Information Table S1 (under "completers") but are also in prior published work. ${ }^{15}$ However, these variables could not be included in the main LGCM analyses, as the present study aimed to examine how a BD diagnosis, compared to $\mathrm{HCs}$, affects changes in cognitive performance over time.

\section{2 | Neuropsychological assessment}

A neuropsychological test battery, akin to our previously published work, ${ }^{4,20}$ was administered. Trained staff administered the neuropsychological tasks at study baseline, and 1 and 5 years after study entry. Training and supervision of test administration was overseen by licensed clinicians (KAR, SAL and DFM). The tests were used to measure cognitive performance in four cognitive domains: auditory memory (verbal learning and memory), visual memory (visuospatial memory), fine motor dexterity, and emotion processing. Neuropsychological tests included: the California Verbal Learning Test-II (CVLT-II), ${ }^{31}$ Rey-Osterrieth Complex Figure Test (RCFT), ${ }^{32}$ Purdue Pegboard, ${ }^{33}$ Emotion Perception Test (EPT), ${ }^{34}$ and the Facial Emotion Perception Test (FEPT). ${ }^{35,36}$

Accordant with our previous work ${ }^{20,37}$ and due to the large number of variables within the neuropsychological tests, we used standard data reduction techniques (principal axis factor analysis) to reduce the tests using conceptually and theoretically categorized variables. ${ }^{38-41}$ First, all scores with negative scale properties were inverted; as a result, lower factor scores reflect poorer performance. Second, a confirmatory factor analysis with oblique rotation was computed with the above variables, consistent with our prior study. ${ }^{20}$ The four latent factors were auditory memory (verbal learning and memory), visual memory (visuospatial memory), fine motor dexterity, and emotion processing. Factor scores were calculated by taking the mean z-score of the cognitive subtests used in computing each latent factor score. The subtests and the reliability of each score are illustrated in Supporting Information Table S2. Briefly, scores from the CVLT-II made up the auditory memory domain, scores from the RCFT made up the visual memory domain, scores from the Purdue Pegboard made up the fine motor dexterity domain, and scores from the EPT and FEPT made up the emotion processing domain. The Wechsler Abbreviated Scale of Intelligence vocabulary subtest ${ }^{42}$ was used to estimate overall verbal intelligence.

\section{3 | Data analysis}

We used IBM SPSS 22 (IBM Corp., Armonk, NY, USA) for univariate and bivariate analyses. For bivariate analysis, we used Pearson or Spearman correlation tests. AMOS $22.0^{43}$ was used to run LGCM, which is a 
particular subtype of structural equation modeling. ${ }^{44}$ AMOS uses full information maximum likelihood (FIML) to handle missing data.

We ran four LGCMs for our four outcomes. First, we ran unconditional LGCMs which only included intercepts and slopes as outcomes, without any covariate. Due to low sample size, we did not include quadratic slope (none of the models with nonlinear slopes converged, due to degree of freedom). Then, we ran four conditional LGCMs for our four outcomes, with BD as the main independent variable, age, gender, and education as covariates, and outcomes as intercept and linear slope. We also included covariance between intercept and linear slope.

In our conditional models, we drew eight paths from BD as well as other covariates (age, gender, and education) to intercept and linear slope. As our focus was on main effect of BD in pooled sample comparison of those with $\mathrm{BD}$ and $\mathrm{HCs}$, we could only include covariates that were common between our groups. As a result, this study did not control for clinical variables that are limited to those with BD, such as type of illness, medications, age of onset, or rapid cycling.

We evaluated the fit of our model based on the comparative fit index ( $\mathrm{CFI}>0.90$ ), the chi-square to degrees of freedom ratio (<4.0), and the root mean squared error of approximation (RMSEA; <0.08). ${ }^{45-}$

${ }^{49}$ An RMSEA value $<0.08$ is generally considered a good fit. ${ }^{45}$ Low sample size explains our RMSEA of $0.08 .^{50}$ In addition, we did not report standardized root mean square residual (SRMR) because of our low sample size. SRMR is considerably biased (positively) for small $\mathrm{N}$ and for low $d f$ studies. ${ }^{50}$ Unstandardized regression coefficients, standard errors (SEs), and $P$-values were reported for each path.

\section{3 | RESULTS}

\section{1 | Descriptive statistics}

As reported in our prior work, ${ }^{15}$ there was a significant group difference for education $(t(105)=2.05, P=.04)$, with the $B D$ group having fewer years of education than the $\mathrm{HC}$ group. There was a trend toward significant group differences in age $(t(105)=-1.94, P=.06)$ but no significant difference in gender $\left(\chi^{2}(1, N=107)=0.03, P=.86\right)$ or general verbal intelligence (vocabulary scaled score) $(t(105)=0.91, P=.37)$. Table 1 illustrates group comparisons for each of the four cognitive performance domains at each of the three time points: baseline, 1 year, and 5 years. The BD group generally underperformed in visual memory and fine motor compared to the $\mathrm{HC}$ group across all three time points.

\section{2 | Bivariate analysis}

Table $2 a$ shows the intercorrelations between the cognitive performance factor scores across the three time points for all participants. Based on the oblique factor scores being related to the same cognitive construct, baseline visual memory, auditory memory, fine motor, and emotion processing scores were positively correlated with each other, with coefficients ranging from .19 to .59. For intercorrelations, visual memory at baseline was significantly and moderately correlated with visual memory year $1(r=.66, P<.001)$ and significantly and strongly correlated with visual memory year $5(r=.77, P<.001)$. Auditory memory at baseline was significantly and moderately correlated with auditory memory year $1(r=.59, P<.001)$ and year $5(r=.66, P<.001)$. Motor at baseline showed significant and strong correlations with motor year 1 $(r=.78, P<.001)$ and year $5(r=.75, P<.001)$. Emotion processing at baseline showed a strong and significant correlation with emotion processing year $1(r=.85, P<.001)$ but a weaker albeit still significant correlation with emotion processing year $5(r=.41, P=.001)$, indicating that this variable was less stable over time.

Age was consistently negatively associated with visual memory, fine motor, and emotion processing at baseline, year 1 , and year 5 . Education was only significantly correlated with visual memory at year 1 , auditory memory at year 5 , and motor at baseline and year 5 (Table 2a). As this study examines cognitive trajectory based on diagnosis instead of the clinical aspects of $\mathrm{BD}$, correlations between these clinical variables and the baseline, year 1 , and year 5 cognitive performance scores are given in Supporting Information Table S3. Chronicity of mood symptoms, impact of illness based on clinician ratings, age of onset, years with $B D$ illness, and number of lifetime mood episodes (all from the baseline DIGS interview) were all consistently negatively associated with the cognitive performance scores. Of those, significant associations were found between chronicity of mood and auditory memory at years 1 and 5 ( $r=-.28,-.31)$, illness impact and emotion processing at baseline, auditory memory and fine motor at year $5(r=-.24,-.26,-.25)$, and age of $\mathrm{BD}$ onset and fine motor at baseline $(r=-.21)$. Years with illness and visual memory at baseline were significantly correlated with those at year 5 , fine motor at years 1 and 5 , and emotion processing at year 5 ( $r$ values ranged from -.25 to -.32 ; see Table S3), and number of lifetime mood episodes and auditory memory at year $1(r=-.32)$. Depression and mania symptom ratings (Table $2 \mathrm{~b}$ ) were not consistently related to cognitive factor scores, although YMRS at year 1 was negatively associated with visual and auditory memory at year 1 ( $r=-.22,-.22)$. In addition, while there were significant correlations between rapid cycling and auditory memory at year $1(r=-.31)$ and history of psychosis and visual memory at baseline ( $r=.23)$, no consistent patterns were observed.

\section{3 | Latent growth curve modeling}

The fit of the visual memory model was very good $(\mathrm{CFI}=0.974$, $\chi^{2}=13.592, d f=8, \chi^{2} / d f=1.699, P=.093$, RMSEA=0.081). According to the results of the model, age was negatively associated with baseline visual memory, suggesting that higher age at baseline is associated with a lower visual memory score. Education was positively associated with baseline visual memory, suggesting that more educated individuals had higher visual memory scores at baseline. There was a positive and significant covariance between the intercept and linear slope of visual memory, suggesting that individuals who are worse off at the start point regarding visual memory would experience a larger decline over time $(B=0.052, S E=0.017, P=.002$; Figure $1 A)$.

The fit of the auditory memory model was also very good ( $\mathrm{CFI}=0.982$, $\chi^{2}=10.832, d f=8, \chi^{2} / d f=1.354, P=.211$, RMSEA=0.057). As Figure $1 \mathrm{~B}$ suggest, education was positively associated with baseline auditory memory score, which suggests that individuals with a high education level had a higher baseline auditory memory level. Age, gender, and 


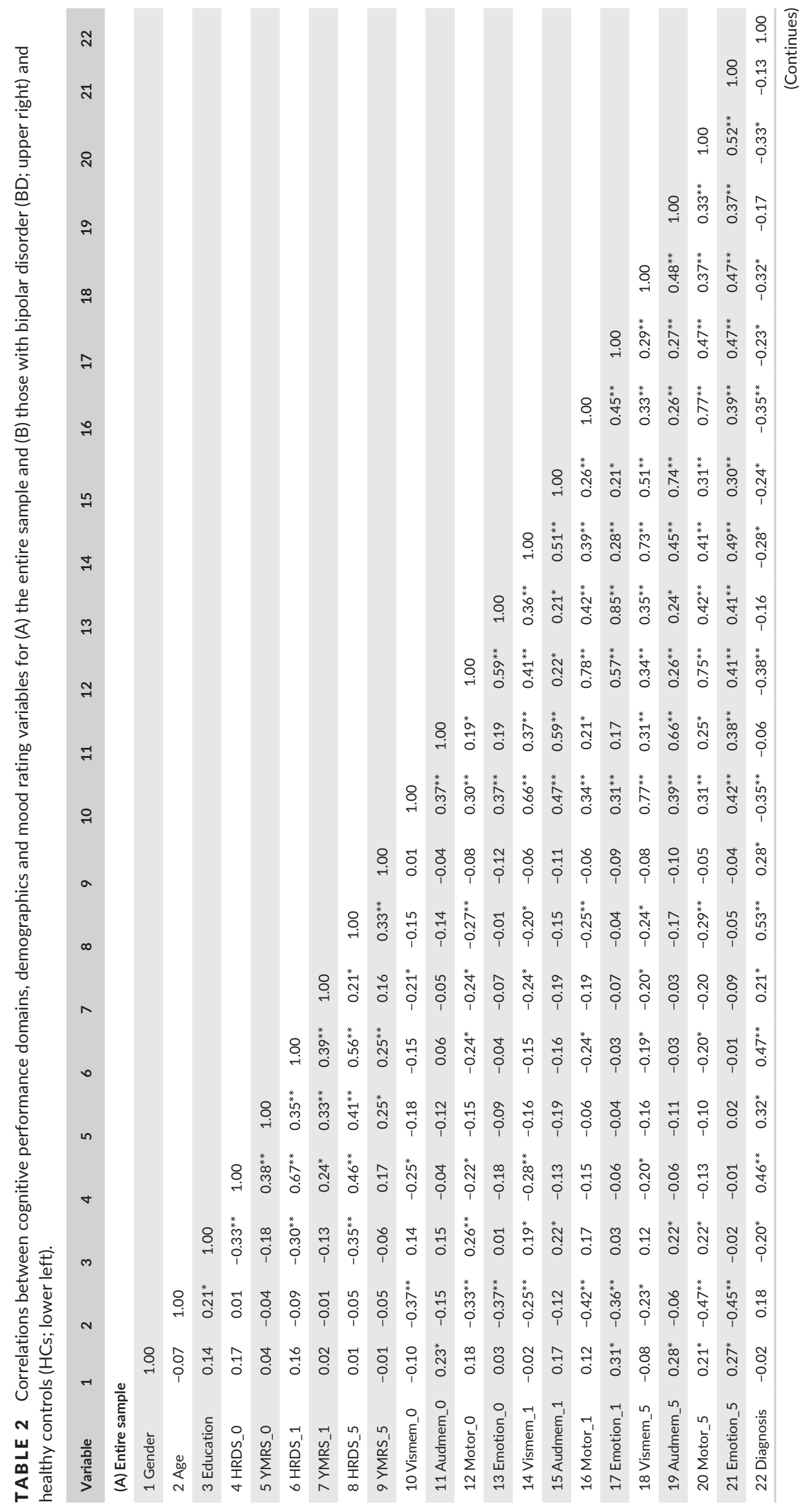




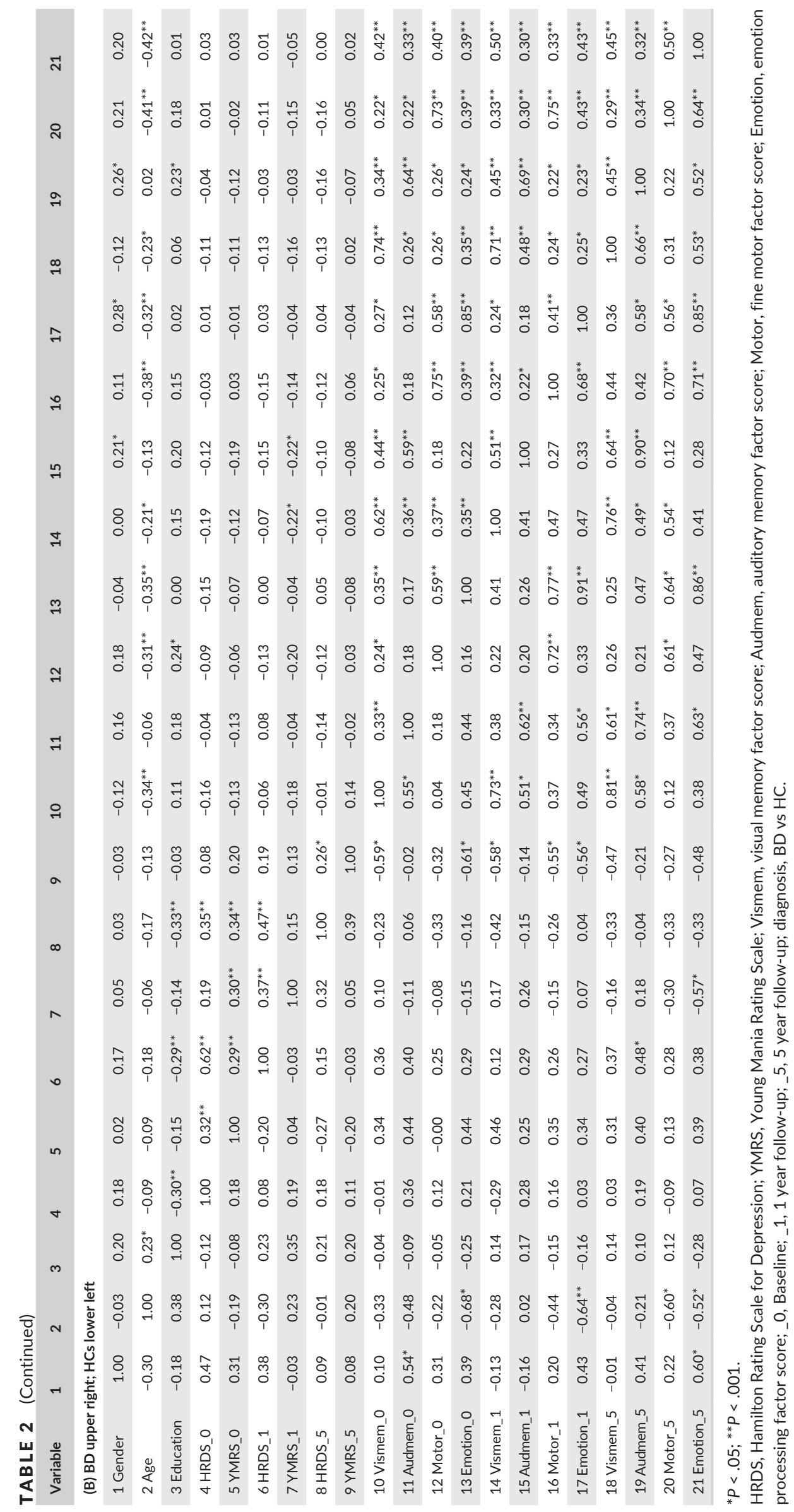


FIGURE 1 Trajectory of cognitive performance over 5 years amongindividuals with bipolar disorder and controls using latent growth modeling. (A) Visual memory (VM), B) Auditory memory (AM), 1C) Emotion processing (EP), 1D) Fine motor dexterity (FM).

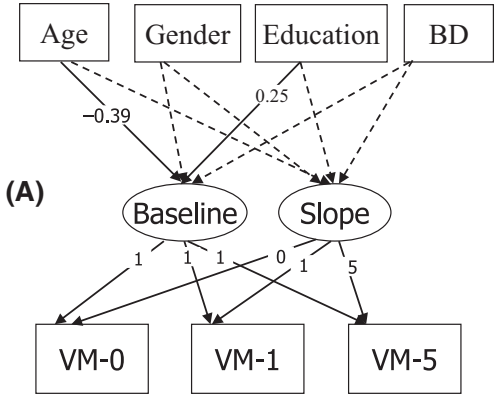

$\mathrm{CFI}=.974$, Chi-square $=13.592, \mathrm{df}=8$, Chi-square $/ \mathrm{df}=1.699$, Probability level $=.093$, RMSEA $=0.081$

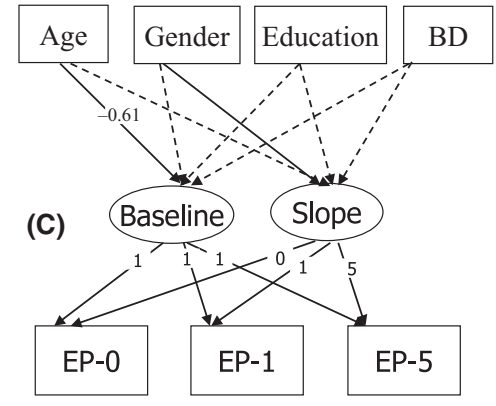

$\mathrm{CFI}=.947$, Chi-square $=15.272, \mathrm{df}=3$, Chi-square $/ \mathrm{df}=2.545$, Probability level .018, RMSEA $=0.046$

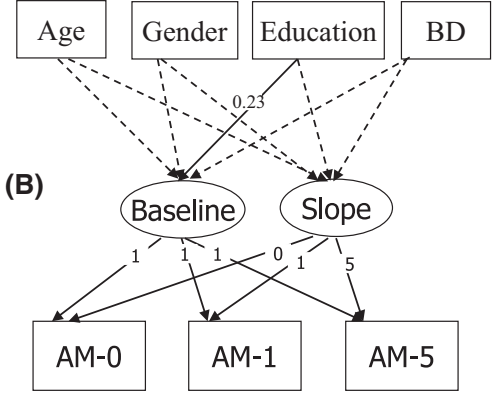

$\mathrm{CFI}=.982$, Chi-square $=10.832, \mathrm{df}=8$, Chi-square $/ \mathrm{df}=1.354$, Probability level $=.211, \mathrm{RMSEA}=.057$

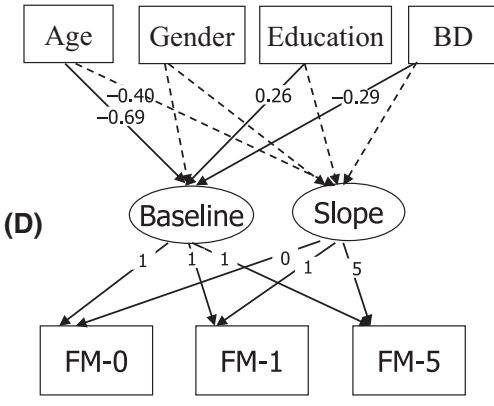

$\mathrm{CFI}=.959$, Chi-square $=17.672, \mathrm{df}=7, \mathrm{Chi}$-square $/ \mathrm{df}=2.525$, Probability level $=.014$, RMSEA $=0.051$
BD were not associated with baseline or change in auditory memory score. There was not any significant covariance between intercept and linear slope of auditory memory, suggesting that the rate of decline over time is independent of the start point for visual memory ( $B=0.016, \mathrm{SE}=0.010, P=.127$ ).

The fit of the emotion processing model was very good (CFI=0.947, $\chi^{2}=15.272, d f=3, \chi^{2} / d f=2.545, P=.018$, RMSEA=0.046). Based on this model, diagnosis (BD vs $\mathrm{HC}$ ) was not significantly associated with intercept or slope of emotion processing. Age was, however, negatively associated with baseline emotion processing score, suggesting that individuals who had a higher age at baseline had a lower emotion processing score at baseline. Female gender was negatively associated with the slope of the emotion processing over the 5-year follow-up, suggesting that female gender was associated with worse emotion processing change during the 5 -year period. Education was not associated with baseline or change of emotion processing. There was a negative and significant covariance between the intercept and linear slope of emotion processing $(B=-0.280, S E=0.054, P<.001)$, suggesting that individuals who start with better emotion processing would be at risk of a larger decline over time (Figure 1C).

The fine motor model also showed a very good fit (CFI=0.959, $\chi^{2}=17.672, d f=7, \chi^{2} / d f=2.525, P=.014$, RMSEA=0.051). As Figure 1D suggest, $B D$ and age were negatively associated and education was positively associated with the intercept of fine motor, suggesting a lower level of fine motor at baseline among older individuals and those with BD compared to HCs. Education was positively associated with baseline fine motor, suggesting individuals with higher education perform better at baseline for fine motor. Age was negatively associated with the fine motor change over time, suggesting that having higher age at baseline was associated with lower fine motor change over time. There was no significant covariance between the intercept and linear slope of fine motor, suggesting that decline of fine motor over 5 years does not depend on its start point $(B=-.003, S E=0.014$, $P=.839)$.

\section{4 | DISCUSSION}

In line with our recently published findings showing poorer executive functioning performance among those with BD compared to controls at baseline and similar linear change in executive functioning, ${ }^{15}$ our current findings show the same pattern when assessing memory, emotion processing, and fine motor dexterity skills. There was no difference in the linear decline of these cognitive scores over time between the BD and $\mathrm{HC}$ samples, similarly suggesting that the longitudinal course of memory, emotion processing, and fine motor dexterity over 5 years is not dependent on having a BD diagnosis. The rate of change in those with $\mathrm{BD}$ was equivalent to that in psychiatrically unaffected individuals.

Further, these results suggest that the cognitive deficits in BD are not age-accelerated, age-compounded, or neurodegenerative, extending earlier work using smaller samples. ${ }^{9-12,51}$ We found a significant effect of age on visual memory, emotion processing, and fine motor dexterity and a significant effect of education on visual and auditory memory and fine motor dexterity such that those who are older at baseline and with less education perform poorer in these cognitive areas, regardless of having a BD diagnosis or no psychiatric diagnosis. This is expected based on normative aging ${ }^{52}$ and protective effects of education. $^{53}$

Age appears to affect change in performance over time for fine motor dexterity, which is also consistent with literature reporting that 
normal aging is associated with changes in processing speed. Our findings indicate that this continues to be the case, regardless of having a $\mathrm{BD}$ diagnosis. Most importantly, having a BD diagnosis does not further enhance or accelerate this change over time, despite those with BD performing worse in fine motor skills compared to the control sample. Having slower fine motor and processing speed skills is a characteristic feature of the BD illness, and thus our results are in line with accepted knowledge.

To address limitations in our prior work focused on executive functioning trajectories, we now highlighted a broader area of cognition to include fine motor dexterity, emotion processing, and memory, all areas thought to be deficient in BD, and memory in particular is argued to be one area of disease-related progressive change. As this is a particular strength in the present study, especially when combined with our prior work on executive functioning, we did not capture other areas that may be less commonly affected in psychiatric or neurological illness, such as social cognition, visual integration, or language functioning. In line with limitations outlined in our prior work, this study also warrants further investigation to address the generalizability of the results. Our HC group was relatively small $(n=17)$ and it is possible that they are not entire representative of a "no diagnosis" group, especially given their younger age, which may influence the overall findings. Along these lines, our overall sample size is not large enough to increase our significance threshold to account for multiple testing and therefore our findings should only be considered preliminary and in need of replication in higher powered samples. Further, we could not control for medication usage as this continues to be a naturalistic study, and we also include controls in our LGCM who were not on any psychotropic medications to begin with. From a methodological perspective, we continue to have too small a sample size to include quadratic slope in addition to linear slope, so we are not able to comment on nonlinear slopes. In our future work, we plan to examine if specific scar or illness burden factors are related to cognitive trajectories using latent growth curve analysis; such analyses and results are too lengthy to include in the current study and warrant their own focused investigation. This will also allow us to include covariates specific to the BD group in our models. Specifically, we note in Supporting Information Table S3 that chronicity of mood symptoms, impact of illness based on clinician ratings, age of onset, years with BD illness, and number of lifetime mood episodes were all consistently negatively associated with the cognitive performance scores in the BD sample. We plan to investigate how these variables influence cognitive trajectories within bipolar illness in a deeper investigation into what illness parameters may influence cognitive decline.

Consistent with findings from our longitudinal cohort study that showed individuals with BD do not appear to have neurodegeneration or age-compounding effects upon executive functioning skills, our current findings show that this may be the case in other areas of cognition, including memory, emotion processing, and fine motor dexterity. Individuals with BD show persistent cognitive deficits compared to controls, but with similar age-related declines across 5 years. We intend to continue to follow this cohort, many of whom are now within their 10th year of follow-up, to determine if BD continues to be more a relapsing-remitting psychiatric illness than a neuroprogressive one. We anticipate that these findings can inform the way in which treatment is managed over time, notably indicating that those with BD may be at risk for cognitive deficits, but are likely not at any great risk for neurodegeneration, at least over a 5-year period.

\section{ACKNOWLEDGEMENTS}

We would like to express appreciation to our research participants in the Prechter Longitudinal Study of Bipolar Disorder. We would also like to acknowledge and thank our research team consisting of Holli Bertram, Christine Brucksch, Brent Doil, Valerie Foster, Laura Gabriel, Nicole Greer, Lauren Grove, Brennan Haase, Gloria Harrington, Alexander Hayek, Michelle Kassel, Marisa Kelly, Katie Lavin, Kortni Meyers, Jennifer Montgomery, Lisa O'Donnell, Philip Presnell and Anne Weldon, and the rest of the staff of the Prechter Bipolar Research Team for their contributions to this project.

\section{DECLARATION OF INTEREST}

Dr. Ryan, Dr. Assari, Ms. Angers, Ms. Babu, Ms. Easter, Ms. Pester, Dr. Marshall, and Dr. Hinrichs report no competing interests. Dr. Langenecker has served as a consultant for Cogstate, Ltd and Easter Seals, Inc., in work unrelated to the present work. Dr. Mclnnis has affiliations with Janssen Pharmaceuticals.

\section{REFERENCES}

1. Martínez-Arán A, Vieta $E$, Reinares $M$, et al. Cognitive function across manic or hypomanic, depressed, and euthymic states in bipolar disorder. Am J Psychiatry. 2004;161:262-270.

2. Clark L, Kempton MJ, Scarnà A, Grasby PM, Goodwin GM. Sustained attention-deficit confirmed in euthymic bipolar disorder but not in first-degree relatives of bipolar patients or euthymic unipolar depression. Biol Psychiat. 2005;57:183-187.

3. El-Badri SM, Ashton $\mathrm{CH}$, Moore PB, Marsh VR, Ferrier IN. Electrophysiological and cognitive function in young euthymic patients with bipolar affective disorder. Bipolar Disord. 2001;3:79-87.

4. Ryan KA, Vederman AC, McFadden EM, et al. Differential executive functioning performance by phase of bipolar disorder. Bipolar Disord. 2012;14:527-536.

5. Porter RJ, Robinson LJ, Malhi GS, Gallagher P. The neurocognitive profile of mood disorders - a review of the evidence and methodological issues. Bipolar Disord. 2015;17:21-40.

6. Goodwin GM, Martinez-Aran A, Glahn DC, Vieta E. Cognitive impairment in bipolar disorder: neurodevelopment or neurodegeneration? An ECNP expert meeting report. Eur Neuropsychopharmacol. 2008;18:787-793.

7. Berk M. Neuroprogression: pathways to progressive brain changes in bipolar disorder. Int J Neuropsychopharmacol. 2009;12:441-445.

8. Martino DJ, Strejilevich SA, Scápola M, et al. Heterogeneity in cognitive functioning among patients with bipolar disorder. J Affect Disord. 2008;109:149-156.

9. Arts B, Jabben N, Krabbendam L, van Os J. A 2-year naturalistic study on cognitive functioning in bipolar disorder. Acta Psychiatr Scand. 2011;123:190-205.

10. Mora E, Portella MJ, Forcada I, Vieta E, Mur M. Persistence of cognitive impairment and its negative impact on psychosocial functioning 
in lithium-treated, euthymic bipolar patients: a 6-year follow-up study. Psychol Med. 2013;43:1187-1196.

11. Burdick KE, Goldberg JF, Harrow M. Neurocognitive dysfunction and psychosocial outcome in patients with bipolar I disorder at 15-year follow-up. Acta Psychiatr Scand. 2010;122:499-506.

12. Balanza-Martinez V, Tabares-Seisdedos R, Selva-Vera G, et al. Persistent cognitive dysfunctions in bipolar I disorder and schizophrenic patients: a 3-year follow-up study. Psychother Psychosom. 2005;74:113-119.

13. Tsitsipa $\mathrm{E}$, Fountoulakis $\mathrm{KN}$. The neurocognitive functioning in bipolar disorder: a systematic review of data. Ann Gen Psychiatry. 2015;14:42.

14. Raust A, Daban C, Cochet B, Henry C, Bellivier F, Scott J. Neurocognitive performance as an endophenotype for bipolar disorder. Front Biosci. 2014;6:89-103.

15. Ryan KA, Assari S, Pester BD, et al. Similar trajectory of executive functioning performance over 5 years among individuals with bipolar disorder and unaffected controls using latent growth modeling. $J$ Affect Disord. 2016;199:87-94.

16. Bora E, Yucel M, Pantelis C. Cognitive endophenotypes of bipolar disorder: A meta-analysis of neuropsychological deficits in euthymic patients and their first-degree relatives. J Affect Disord. 2009;113:1-20.

17. Mann-Wrobel MC, Carreno JT, Dickinson D. Meta-analysis of neuropsychological functioning in euthymic bipolar disorder: an update and investigation of moderator variables. Bipolar Disord. 2011;13:334-342.

18. Robinson LJ, Ferrier IN. Evolution of cognitive impairment in bipolar disorder: a systematic review of cross-sectional evidence. Bipolar Disord. 2006;8:103-116.

19. Quraishi S, Frangou S. Neuropsychology of bipolar disorder: a review. J Affect Disord. 2002;72:209-226.

20. Langenecker SA, Saunders EFH, Kade AM, Ransom MT, McInnis MG. Intermediate: cognitive phenotypes in bipolar disorder. J Affect Disord. 2010;122:285-293.

21. Curran PJ, Obeidat K, Losardo D. Twelve frequently asked questions about growth curve modeling. J Cogn Dev. 2010;11:121-136.

22. Granholm AC, Boger H, Emborg ME. Mood, memory and movement: an age-related neurodegenerative complex? Curr Aging Sci. 2008;1: 133-139.

23. Nurnberger JI Jr, Blehar MC, Kaufmann CA, et al. Diagnostic interview for genetic studies. Rationale, unique features, and training. NIMH Genetics Initiative. Arch Gen Psychiatry. 1994;51:849-859; discussion 63-4.

24. Hamilton MAX. Development of a rating scale for primary depressive illness. Br J Soc Clin Psychol. 1967;6:278-296.

25. Young RC, Biggs JT, Ziegler VE, Meyer DA. A rating scale for mania: reliability, validity and sensitivity. Br J Psychiatry. 1978;133:429-435.

26. Almeida JRC, Akkal D, Hassel S, et al. Reduced gray matter volume in ventral prefrontal cortex but not amygdala in bipolar disorder: significant effects of gender and trait anxiety. Psychiatry Res. 2009;171:54-68.

27. Davis JM, Chen N. Dose response and dose equivalence of antipsychotics. J Clin Psychopharmacol. 2004;24:192-208.

28. Hassel S, Almeida JRC, Kerr N, et al. Elevated striatal and decreased dorsolateral prefrontal cortical activity in response to emotional stimuli in euthymic bipolar disorder: no associations with psychotropic medication load. Bipolar Disord. 2008;10:916-927.

29. Sackeim HA. The definition and meaning of treatment-resistant depression. J Clin Psychiatry. 2001;62:10-17.

30. Sackeim HA. The definition and meaning of treatment-resistant depression. J Clin Psychiatry. 2001;62(Suppl 16):10-17.

31. Delis D, Kaplan E, Kramer J, Ober BA. California Verbal Learning Test-II. San Antonio, TX: The Psychological Corporation; 2000.

32. Meyers J, Meyers K. Rey Complex Figure and Recognition Trial: Professional Manual. Odessa, FL: Psychological Assessment Resources; 1995.

33. Tiffin J, Asher EJ. The Purdue pegboard: norms and studies of reliability and validity. J Appl Psychol. 1948;32:234-247.
34. Green PW, Allen LM. The Emotional Perception Test. Durham, NC: CogniSyst Inc.; 1997.

35. Rapport LJ, Friedman SR, Tzelepis A, Van Voorhis A. Experienced emotion and affect recognition in adult attention-deficit hyperactivity disorder. Neuropsychology. 2002;16:102-110.

36. Langenecker SA, Bieliauskas LA, Rapport LJ, Zubieta J-K, Wilde EA, Berent $S$. Face emotion perception and executive functioning deficits in depression. J Clin Exp Neuropsychol. 2005;27:320-333.

37. Ryan KA, Vederman AC, Kamali M, et al. Emotion perception and executive functioning predict work status in euthymic bipolar disorder. Psychiatry Res. 2013;210:472-478.

38. Langenecker SA, Caveney AF, Giordani B, et al. The sensitivity and psychometric properties of a brief computer-based cognitive screening battery in a depression clinic. Psychiatry Res. 2007;152:143-154.

39. Bilder RM, Volavka J, Czobor P, et al. Neurocognitive correlates of the COMT Val (158) met polymorphism in chronic schizophrenia. Biol Psychiat. 2002;52:701-707.

40. Rund BR, Sundet K, Asbjornsen A, et al. Neuropsychological test profiles in schizophrenia and non-psychotic depression. Acta Psychiatr Scand. 2006;113:350-359.

41. Bleiberg J, Kane R, Reeves D, Garmoe WS, Halpern E. Factor analysis of computerized and traditional tests used in mild brain injury research. Clin Neuropsychol. 2000;14:287-294.

42. Wechsler D. Wechsler Abbreviated Scale of Intelligence (WASI) Manual. San Antonio, TX: The Psychological Corporation; 1999.

43. Arbuckle JL. Amos. 8.0 edn. Chicago: SPSS; 2009.

44. Kline RB. Principles and Practice of Structural Equation Modeling. New York, NY: Guilford Press; 2011.

45. Lt H, Bentler PM. Cutoff criteria for fit indexes in covariance structure analysis: conventional criteria versus new alternatives. Struct Eq Model Multi J. 1999;6:1-55.

46. Lei M, Lomax RG. The effect of varying degrees of nonnormality in structural equation modeling. Struct Eq Model Multi J. 2005;12:1-27.

47. Tabachnick BG, Fidell LS. Using Multivariate Statistics, 3rd edn. New York, NY: HarperCollins College Publishers; 1996.

48. Schumacker RE, Lomax RG. A Beginner's Guide to Structural Equation Modeling. Mahwah, NJ: Lawrence Erlbaum Associates; 2004.

49. Bollen KA, Long JS. Testing Structural Equation Models. Thousand Oaks, CA: SAGE Publications; 1993.

50. Kenny DA. 2015. Measuring model fit. https://davidakenny.net/cm/ fit.htm. Accessed May 19, 2017.

51. Xu G, Lin K, Rao D, et al. Neuropsychological performance in bipolar I, bipolar II and unipolar depression patients: a longitudinal, naturalistic study. J Affect Disord. 2012;136:328-339.

52. Salthouse T. The processing speed theory of adult age differences in cognition. Psychol Rev. 1996;103:403-428.

53. Stern Y, Habeck C, Moeller J, et al. Brain networks associated with cognitive reserve in healthy young and old adults. Cereb Cortex. 2005;15:394-402.

\section{SUPPORTING INFORMATION}

Additional Supporting Information may be found online in the supporting information tab for this article.

How to cite this article: Ryan KA, Assari S, Angers K, et al. Equivalent linear change in cognition between individuals with bipolar disorder and healthy controls over 5 years. Bipolar Disord. 2017;19:689-697. https://doi.org/10.1111/bdi.12532 